Volume 8, No.3, May - June 2019

International Journal of Advanced Trends in Computer Science and Engineering

Available Online at http://www.warse.org/IJATCSE/static/pdf/file/ijatcse56832019.pdf

https://doi.org/10.30534/ijatcse/2019/56832019

\title{
Recursive Least Square and Least Means Square Equalizers Optimization Algorithms in Rayleigh Fading
}

\author{
Arianne Grace Dela Cruz, Camilo Cajayon Jr., Joseph Jay Luna, Cris Edward Tomboc \\ De La Salle University, Manila \\ 2401 Taft Ave., Malate, Manila 1004, \\ Philippines, arianne_delacruz@dlsu.edu.ph
}

\begin{abstract}
Recursive Least Squares (RLS) are adaptive filters that search for the coefficient weights that are set to minimize the weighted linear least square cost function of the signal that is inputted. In the RLS derivation, the input signals are known to be deterministic. This method provides fast convergence but its drawback is the high cost of computational complexity. On the other hand, the Least Means Square algorithm is used to mimic the desired filter by searching for its filter coefficients which relate to producing the least means square of the error signal. This method uses a stochastic gradient descent method in the filter. This research will develop a Recursive Least Square and Least Means Square Equalizers Optimization Algorithms in Rayleigh Fading. Testing of the system will be done by using the Matlab Simulink.
\end{abstract}

Key words : Recursive Least Square, Least Means Square, Rayleigh Fading, System Optimization.

\section{INTRODUCTION}

The Least Means Square (LMS) Filter is kind of an Adaptive filter which uses a feedback system and has a Finite Impulse Response (FIR) $[1,2,3]$. If an input $\mathrm{f}(\mathrm{n})$ is passed through the filter, the output produced is a sequence denoted as $y(n)$. For a LMS filter with length $M$, it has coefficients $\{$ b_0,b_1,b_2,b_3,b_4,.........,b_(M-1) $\}$. The desired response denoted as $d(n)$ is then subtracted with the output in order to produce the error e(n) [4]. The system is an adaptive filter, it is designed to handle a broad input [5]. Mathematically,

$$
e(n)=d(n)-y(n)
$$

At each step size the value of the coefficients $b \_k$ is changed depending on the error. The diagram of such syastem would look like

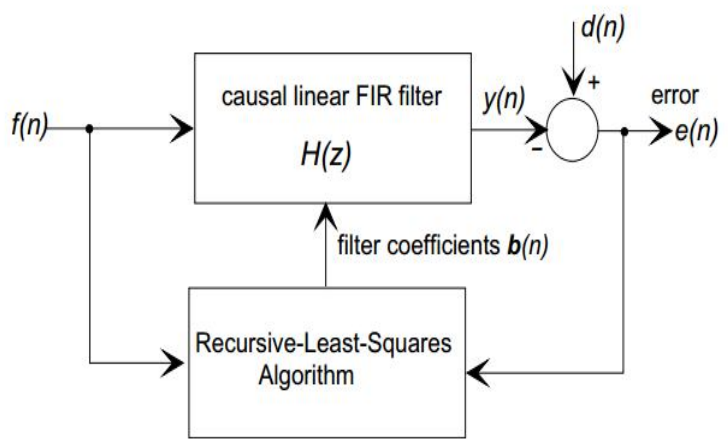

Figure 1: Block Diagram for an LMS Adaptive Filter

As the name suggests, it operates by minimizing the mean square error $\mathrm{E}\left\{\mathrm{e}^{\wedge} 2(\mathrm{n})\right\}$. In order to come up with the algorithm for such a method, we define $J(n)$ as the square performance index. Mathematically the Square Performance Index is:

$$
J(n)=e^{2}(n)=(d(n)-y(n))^{2}=\left(d(n)-\sum_{k=0}^{M-1} b(k) f(n-k)\right)^{2}
$$

In order to reduce $J(n)$, each of the coefficients $b \_k(n)$ is then moved by an amount which is proportional to $(\partial \mathrm{J}(\mathrm{n})) /(\partial \mathrm{b}(\mathrm{k}))$. The step size is defined by a parameter $\Lambda(\mathrm{n})$.Mathematically the Step Size Equation for a Parameter is:

$$
b_{k}(n+1)=b_{k}(n)-\Lambda(n) \frac{\partial J(n)}{\partial b_{k}(n)}, k=0,1,2 \ldots . M-1
$$

And the algorithm for the filter is:

$$
b_{k}(n+1)=b_{k}(n)+\Lambda \rho(n) f(n-k)_{k} k=0,1,2, M-1
$$

The direct form for the LMS filter with length $\mathrm{M}$ is 


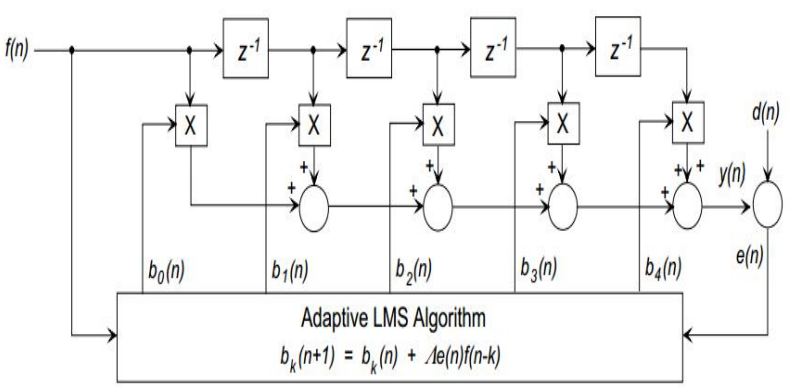

Figure 2: LMS Filter Block Diagram

The advantage of the LMS is that it is simple to implement. It works for many applications as long as the parameters $(\Lambda(n)$ and $\mathrm{M}$ ) are chosen properly. The LMS has its foundations in Spatial Imaging and Neural Networks $[6,7,8]$

The Recursive Least Square (RLS) Filter is also a kind of an Adaptive filter which uses a feedback system and has a Finite Impulse Response (FIR) [9, 10, 11]. Similar to LMS, if an input $\mathrm{f}(\mathrm{n})$ is passed through the filter, the output produced is also a sequence denoted as $y(n)$. For a RLS filter with length M, it has coefficients $\{$ b_0,b_1,b_2,b_3,b_4,.........,b_(M-1).$\quad$ The desired response denoted as $d(n)$ is then subtracted with the output in order to produce the error e(n) [5]. Mathematically the Error Equation for the Desired Response in RLS is:

$$
e(n)=d(n)-y(n)
$$

The diagram of such a system would look like

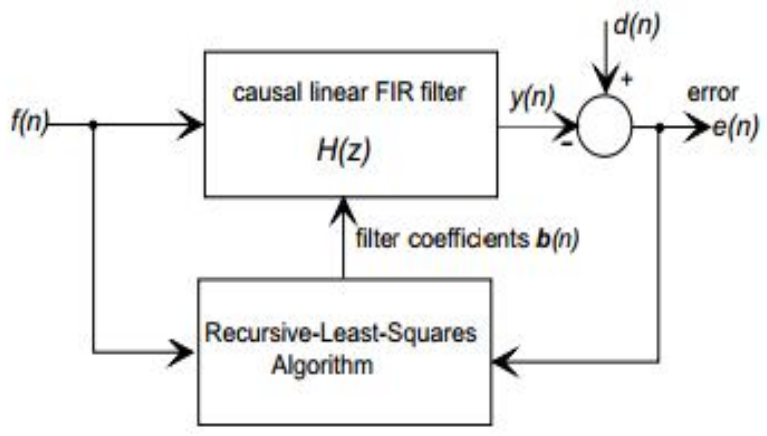

Figure 3: Block Diagram for an RLS Adaptive Filter

It is similar to LMS, except that the coefficients b_k (n) is updated differently $[12,13]$. The RLS filter converges faster than LMS in most conditions, but it has a more complex algorithm and it is harder to implement. For ambiguous data outputs the Rough Set Theory can be used $[14,15,16]$.

In the project, the researchers will attempt to simulate both RLS and LMS using SIMULINK. The researchers will then determine the performance of both systems in Rayleigh Fading and compare them. The database configuration will be like in the researches of $[17,18]$. Matlab Simulink will be used for the simulation [19]. File transfer can be done either by network or usb [20].

\section{DATA AND RESULTS}

The whole block diagram of the system was divided into three parts. The first part is for the RLS equalizer while the second part is the LMS equalizer. The equalizers are different from each other, so the researchers wanted to compare their performance. The last part of the system is a summing point for comparison of the performances of the equalizers.

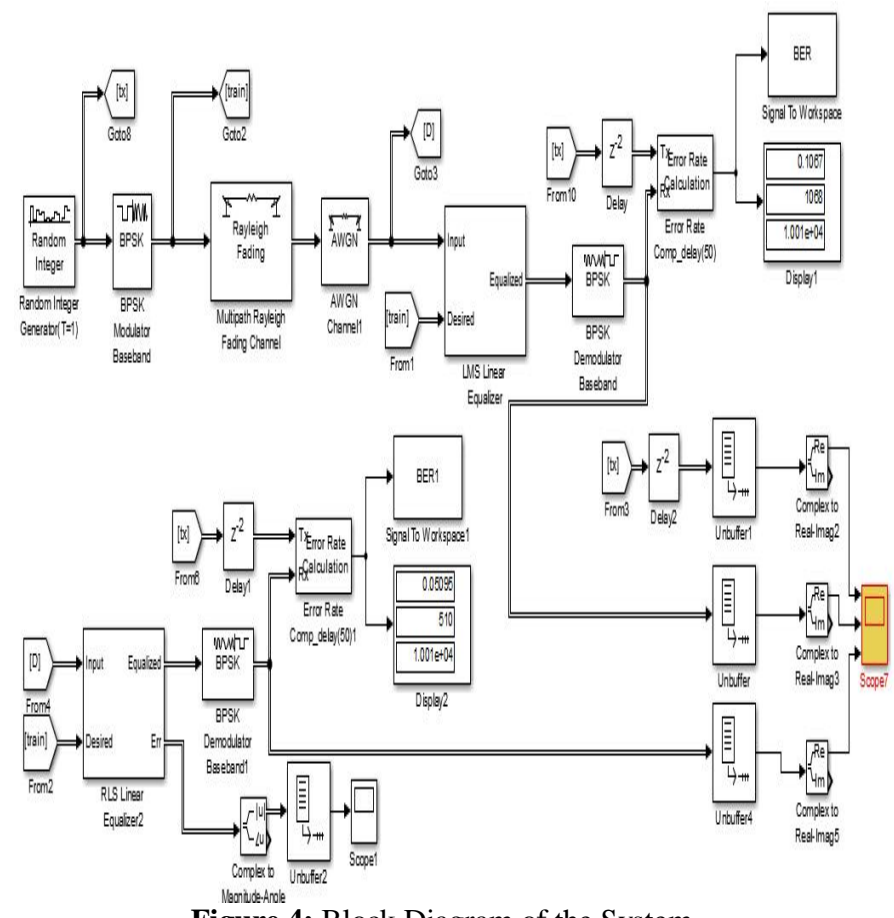

Figure 4: Block Diagram of the System

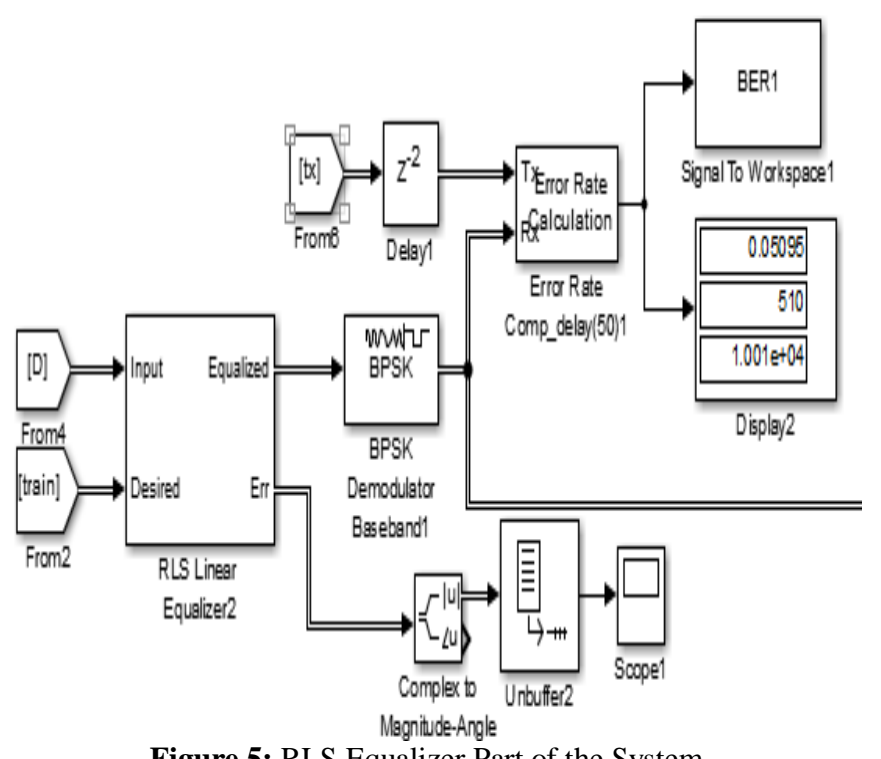

Figure 5: RLS Equalizer Part of the System 


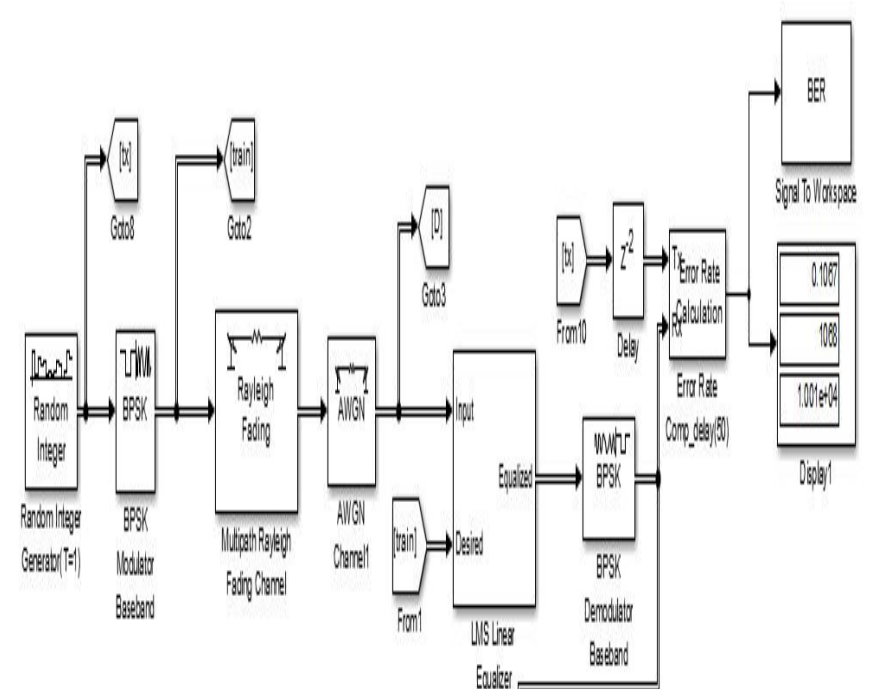

Figure 6: LMS Equalizer Part of the System

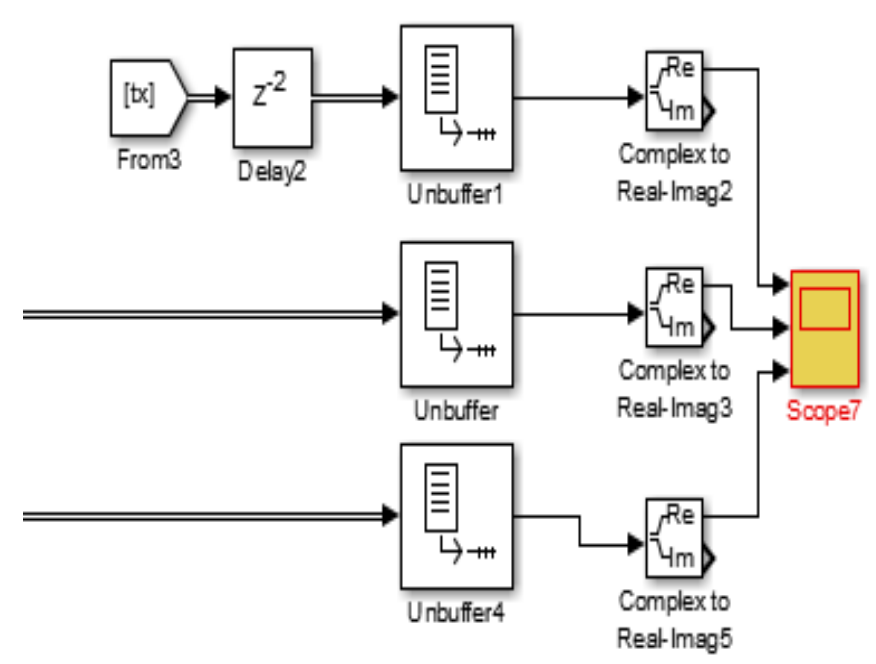

Figure 7: Summing Point of the System for Comparison

It is known in general that RLS (Recursive Least Squares) performs better than LMS (Least Mean Squares) in general. So to prove that this is true, we calculated and compared their BER (bit error rate) using Simulink.

Table 1: Comparing the BER of RLS and LMS Equalizers

\begin{tabular}{|c|c|c|c|}
\hline \multicolumn{2}{|c|}{ RLS BER } & \multicolumn{2}{|c|}{ LMS BER } \\
\hline \multirow{4}{*}{$\rightarrow$} & 0.05095 & & 0.1067 \\
\hline & 510 & $\rightarrow$ & 1068 \\
\hline & $1.001 \mathrm{e}+04$ & & $1.001 e+04$ \\
\hline & Display2 & & Display1 \\
\hline
\end{tabular}

higher than RLS. In conclusion, the RLS performs better than LMS as it has a lower bit error rate.

When it comes to its synchronization, LMS and RLS will not be synchronized initially. It will only be synchronized when the $\mathrm{Eb} / \mathrm{No}$ value is high enough for the performance errors to be negligible already within a time frame. In our case, the system was designed with no multipath and we chose an $\mathrm{Eb} / \mathrm{No}$ of $10 \mathrm{~dB}$ which is considered high already. In Simulink, we compared 3 waveforms. The first waveform consists of the random integer generator delayed by 2 seconds. The second waveform is the LMS equalizer while the last waveform comes from the RLS equalizer. Both equalizer output comes from BPSK demodulator output.

A Scope7

刍
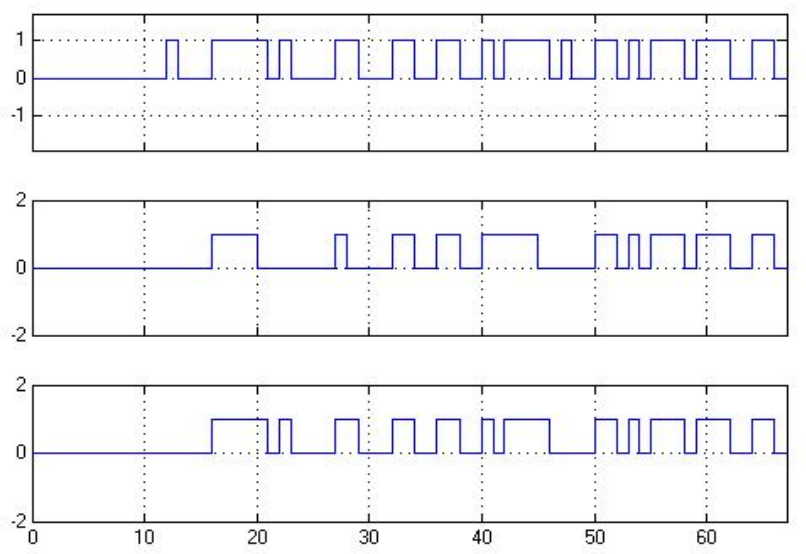

Time offset: 0

Figure 8: Synchronization of the Equalizers using Eb/No of 0.5 $\mathrm{dB}$

Scope7

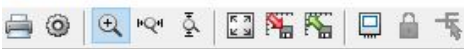
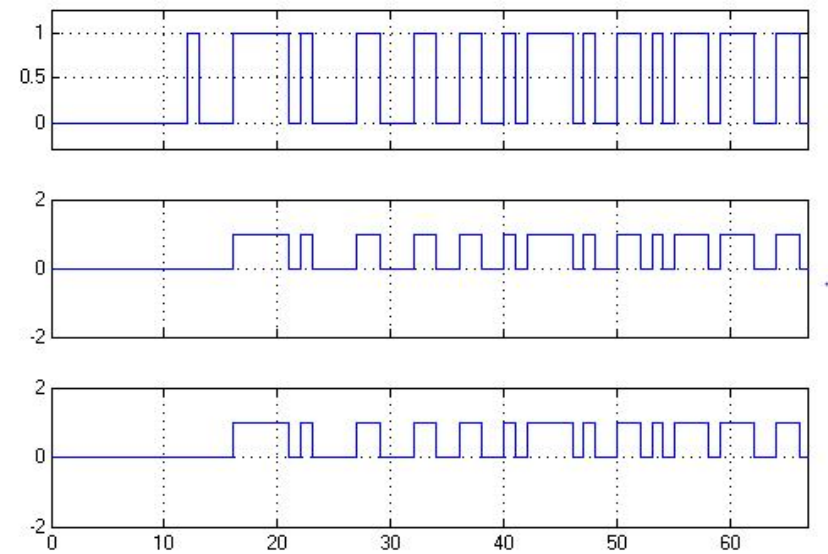

Time offset: 0

Figure 9: Synchronization of the Equalizers using Eb/No of 10 $\mathrm{dB}$

We can see from Table 1 that the bit error rate of LMS is 
From the simulation of 0 to 65 seconds, we can see that the equalizers were not synchronized with the delayed output initially. This may mean that both of them experienced errors at the start of the simulation. With $\mathrm{Eb} / \mathrm{No}$ of $0.5 \mathrm{~dB}$, the second waveform (LMS equalizer) did not synchronize immediately together with the RLS system. With an Eb/No of $10 \mathrm{~dB}$, it is at 15 seconds that the waveforms of the 3 outputs were synchronized with each other. This means that with an $\mathrm{Eb} / \mathrm{No}$ of $10 \mathrm{~dB}$ with results into the equalizers converging and synchronizing at a faster rate compared to when using a low $\mathrm{Eb} / \mathrm{No}$.
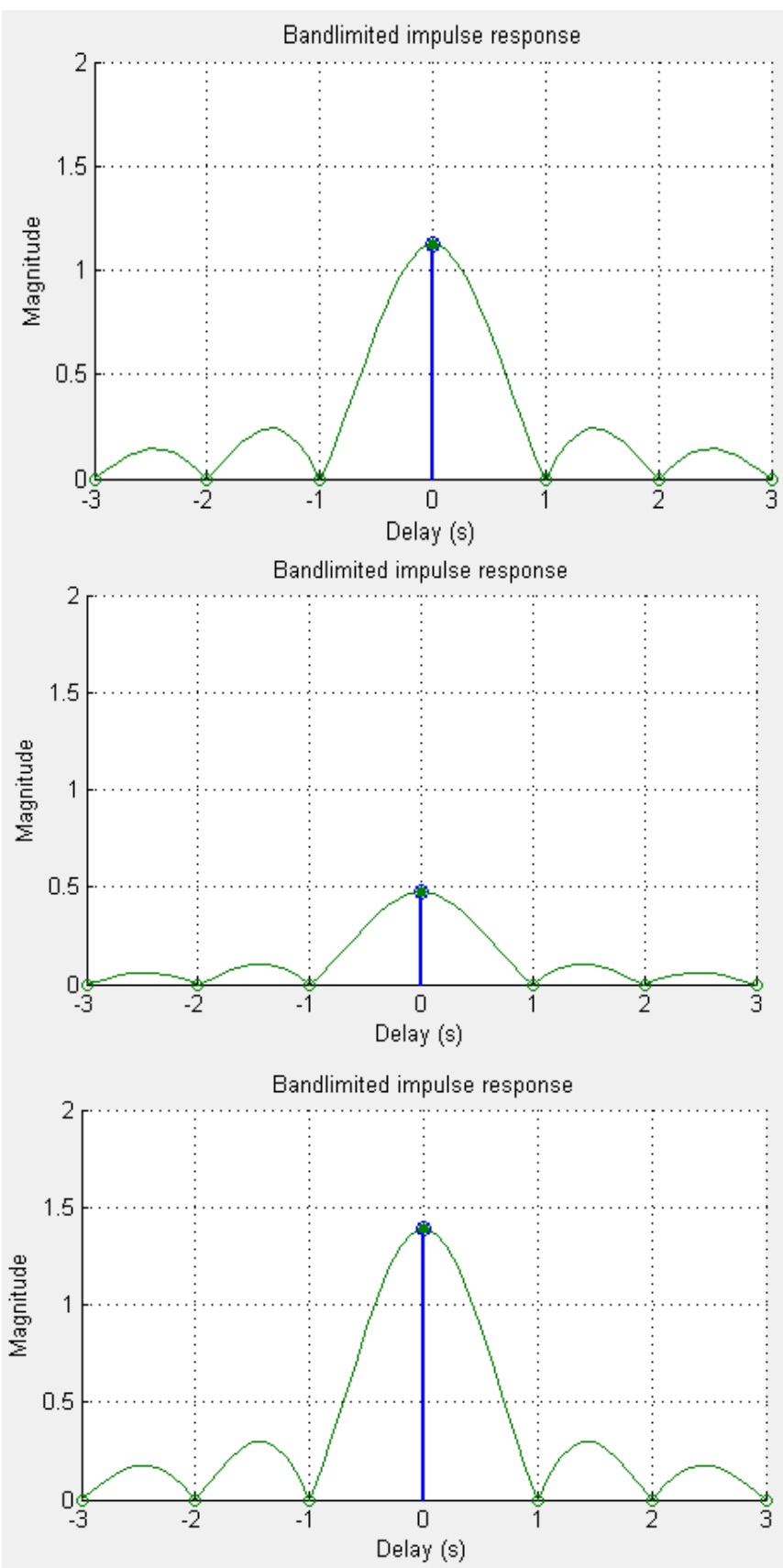

Figure 10: Different Magnitudes of the Impulse Response at 500s, 5000 s, and 10000 s
It can be noticed from the magnitudes that the impulse response changes and varies over time.

\section{ANALYSIS OF DATA}

These two systems were constructed such that they could be compared. The first equalizer considered by the researchers was the Recursive Least Square (RLS) equalizer. The RLS equalizer utilizes the concept of finding the minimum coefficient of a cost function in a recursive manner. It is known to be adaptive. The second equalizer used was the Least Mean Square (LMS) equalizer. Unlike the RLS, LMS uses the concept of the least mean square of a signal. It is also recursive and adaptive like the RLS.

It is important to take note that the system utilized a Rayleigh fading channel. This was unlike the usual channels that the researchers have been exposed to. The Rayleigh channel was used because it would help the researchers to see the behavior of both equalizers if a large number of reflections are present [21]. By doing so, the researchers could see how both equalizers would react to the sudden occurrence of multipath [22]. However, the researchers have limited the project into a system that would not include multipath [23]. The researchers were able to determine the necessary blocks needed in order to compare the RLS and LMS equalizers. The block diagrams for each equalizer was set up and scope was used in order to see their output. By observing their bit error rates, the researchers were able to see that LMS produced a higher bit error rate as compared to RLS. In addition to this, the Eb/No VS BER plot was observed. There are instances where the data that is generated has incomplete information. For this instance, the Rough Set Theory can be used [24, 25].

The plots enabled the researchers to see that a linear graph for the LMS equalizer while the RLS produced an output in line with the theoretical one found in the literature. In addition, the synchronization of the system was observed. Based on the project, the Eb/No could actually influence the speed of synchronization of a system. The researchers were able to see that at a higher $\mathrm{Eb} / \mathrm{No}$, the waveforms synchronized in about 15 seconds. For those errors the Logic Scoring of Preference (LSP) algorithm can be used to optimize the data [26].

\section{LITERATURE REVIEW}

The paper of the 3 authors from India talks about adaptive filter design that is based on different Recursive Least Squares (RLS) and Least Mean Square (LMS) methods. The filter design is applicable to different signals for reduction of noise in waveforms like chirp, saw-tooth, and sinusoidal. To be able to see and analyze the performance of the designed filter, the researchers measured some factors like the MMSE (Minimum mean square error) and the Rate of convergence. All these, ranging from the design up to the simulations, were done 
through the MATLAB software. As expected, the RLS filter shows a better performance compared to the other 2 methods. It showed a better convergence rate, but it has a low minimum mean square error. Also, the output was not as smooth in the chirp and sinusoidal signals as compared with the output using saw-tooth waveform [27].

This next journal is about different adaptive filters again but this time it is for ECG signal processing. The authors are from the Indian Institute of Technology. They decided to make a research on ECG because of the problem that is posted. This problem is the separation of different signals that comes from the power lines, outside electromagnetic fields, and the random movements from the patient's body. For unknown values the Rough Set Theory can be used [28]. Many types of filters are used to be able to remove unwanted signals. So to be able to remove signals that are difficult to remove, the adaptive filter technique is used. The adaptive filter techniques used in the paper were the RLS and LMS filter algorithms, which are the most commonly used filter algorithms. The researchers used Base Line Interference and PLI to be able to remove those signals. The software that they used is MATLAB. The researchers still used filters like LMS and RLS. The output is that the RLS filter shows a much more filtered signal compared to the LMS [29].
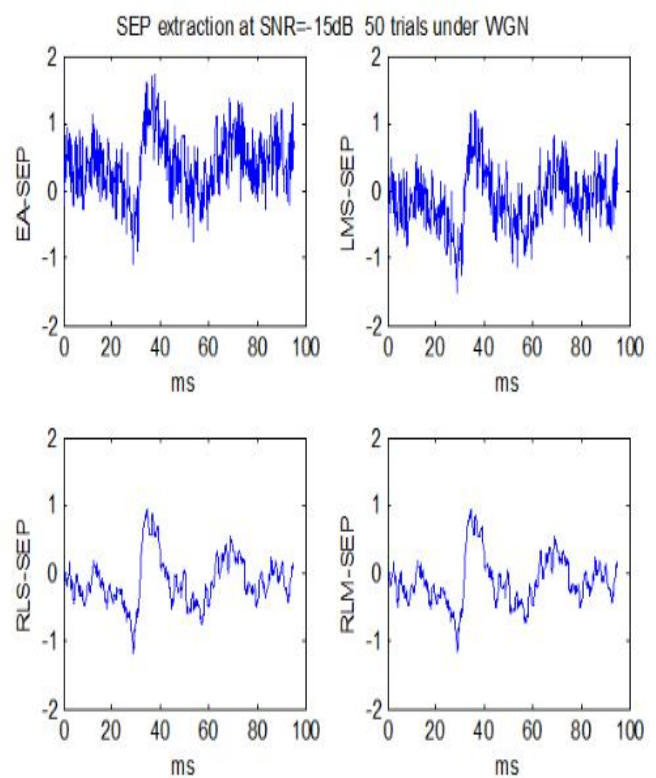

Figure 11: RLS and LMS Output after Filtering

This next paper is similar to the first journal but it compares more filter algorithms. The algorithms that were analyzed here are (1) least mean square (LMS), (2) Time-Varying LMS (TVLMS), (3) Normalized LMS (NLMS), (4) Fast Transversal Recursive Least Squares (FTRLS), and (5) Recursive Least Squares (RLS). The researchers are concerned with the Signal to Noise Ratio (SNR) and the complexity of the computations regarding the process. It was known that these adaptive filtering algorithms have the convergence speed and complexity dependable with each other and that the LMS filter algorithm performs poorly compared to the RLS filter algorithm. With a sampling rate of $1.5 \mathrm{KHz}$, the researchers varied the noise variance and compared how the SNR was improved in varying noise variance. SNR was improved when noise variance becomes lower. This is because the signal is more evident when the noise is low. It was seen that the SNR of LMS degraded higher compared to the degradation of the SNR of NLMS and RLS when the noise is increased [30]. The collected data must also be optimized in a database [31,32].

This time, the paper focuses on the RLS filter alone as an adaptive noise canceller. The concept of adaptive noise canceling is a method of estimating signals that are disruptive due to different additive noise. The noise cancellation was done through the RLS for the noise to be removed and attenuated from the original signal. A noise filter block was used to remove the signals while the original signals were directed on the desired port. It uses a sine wave as its original signal. The authors used Simulink for its simulation and design and the output was not restored $100 \%$ from the original one. This means that the system encountered errors in its simulation. The RLS converges faster than LMS but using RLS requires more complex computations as mentioned in the paper [33].

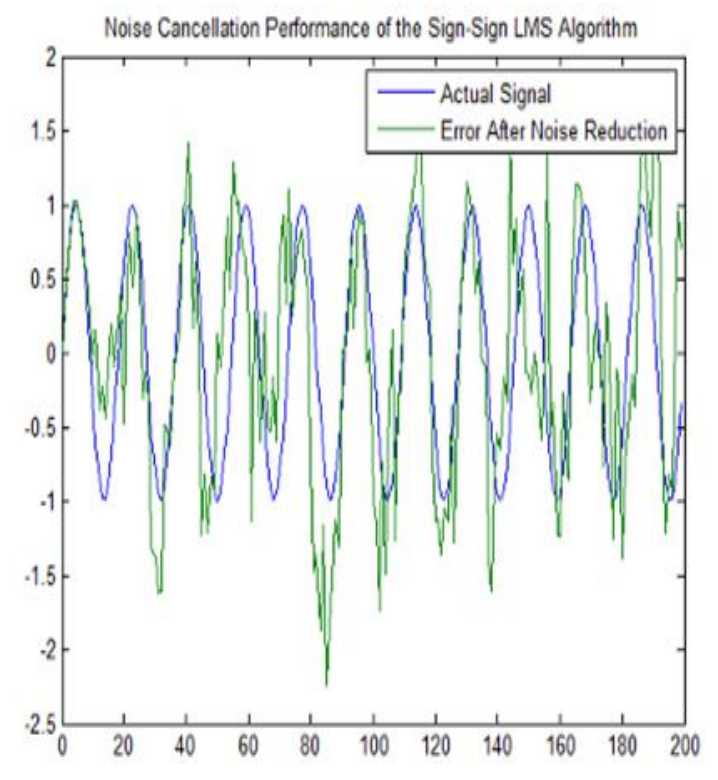

Figure 12: Noise Cancellation Method

The last journal proposes a new relationship between the error signal and the step factor. The relationship between the 2 factors would be able to address some problems in the filter such as performance and convergence time. A new algorithm was also presented to be able to address the inconsistency among the factors such as error performance and time of converging. Also, an improved algorithm for the step-size LMS algorithm was introduced. The step-size LMS adaptive 
filter is a filter with a bigger initial step-size for a faster convergence speed. A bigger initial step size would allow the noise signals to be avoided to reduce the error in the system. As the step-size moves away from the initial one, the step size is reduced. This is done so that the static error will be reduced. The conclusion of the authors is that these algorithms are expected to be improved and these will be widely used in signal processing, control systems, and in the field on communications [34].

\section{CONCLUSION}

Equalizers are essential in order to eliminate noise and interference in a system. These equalizers could be classified as being either linear or non-linear. For the summary of the course, the researchers decided to utilize two equalizers and observe their differences. Basically, the project shows a comparison between the said equalizers.

This research introduced the concept of the Rayleigh channel. The purpose of the project was to determine the error detecting capabilities of the equalizer, having this channel would help the researchers in effectively accomplishing the task. In addition, the Additive White Gaussian Noise (AWGN) channel was also utilized in the design. The RLS equalizer block and the LMS equalizer block that was used by the researchers were already in Simulink. The two blocks were modulated using Binary Phase Shift Keying (BPSK). With the use of the bit error rate calculator, the researchers were able to see that the RLS equalizer produced a lower bit error rate. Thus, the RLS equalizer performs better than the LMS equalizer in terms of the errors detected before transmission. In terms of the synchronization of the equalizers, the researchers initially chose a $5 \mathrm{~dB} \mathrm{~Eb} / \mathrm{No}$. By observation, the researchers were able to see that both of them were not synchronized as expected. As a result, the researchers optimized the $\mathrm{Eb} / \mathrm{No}$ level to $10 \mathrm{~dB}$. From the waveform obtained, the researchers concluded that the waveforms were indeed synchronized, and it occurred at a fast rate.

\section{REFERENCES}

[1] T. Woo, "Fast hierarchical least mean square algorithm," IEEE Signal Processing Letters. Vol. 8, No. 11, pp. 289-291, 2001. https://doi.org/10.1109/97.969446

[2] E. Walach and B. Widrow, "The least mean fourth (LMF) adaptive algorithm and its family," IEEE Transactions on Information Theory. Vol. 30, No. 2, pp. 275-283, 1984. https://doi.org/10.1109/TIT.1984.1056886

[3] Y. Li and M. Hamamura, "An Improved Proportionate Normalized Least-Mean-Square Algorithm for Broadband Multipath Channel Estimation," The Scientific World Journal. Vol. 2014, 2014. https://doi.org/10.1155/2014/572969
[4] D. Cartes, L. Ray, and R. Collier, "Experimental evaluation of leaky least-mean-square algorithms for active noise reduction in communication headsets," The Journal of the Acoustical Society of America. Vol. 111, 2002. https://doi.org/10.1121/1.1448314

[5] MIT OpenCourseWare. Signal Processing: Continuous and Discrete. Retrieved from http://ocw.mit.edu/courses/mechanical-engineering/2-16 1-signal-processing-continuous-and-discrete-fall-2008/st udy-materials/rls.pdf. 2008.

[6] P. Loresco and A. Africa, "ECG Print-out Features Extraction Using Spatial-Oriented Image Processing Techniques." Journal of Telecommunication, Electronic and Computer Engineering. Vol. 10, Nos. 1-5, pp. 15-20, 2018.

[7] S. Brucal, A. Africa, and E. Dadios, "Female Voice Recognition using Artificial Neural Networks and MATLAB Voicebox Toolbox." Journal of Telecommunication, Electronic and Computer Engineering. Vol. 10, Nos. 1-4, pp. 133-138, 2018.

[8] A. Africa and J. Velasco, "Development of a Urine Strip Analyzer using Artificial Neural Network using an Android Phone." ARPN Journal of Engineering and Applied Sciences. Vol. 12, No. 6, pp. 1706-1712, 2017.

[9] W. Zheng, D. Dan, W. Cheng, and Y. Xia, "Real-time dynamic displacement monitoring with double integration of acceleration based on recursive least squares method." Measurement. Vol. 141, pp. 460-471, 2019.

https://doi.org/10.1016/j.measurement.2019.04.053

[10] C. Elisei-Iliescu, C. Stanciu, C. Paleologu, J. Benesty, C. Anghel, and S. Ciochină, "Efficient recursive least-squares algorithms for the identification of bilinear forms.” Digital Signal Processing. Vol. 83, pp. 280-296, 2018. https://doi.org/10.1016/j.dsp.2018.09.005

[11] H. Kasai, "Fast online low-rank tensor subspace tracking by $\mathrm{CP}$ decomposition using recursive least squares from incomplete observations." Neurocomputing. Vol. 347, pp. 177-190, 2019. https://doi.org/10.1016/j.neucom.2018.11.030

[12] T. Gush, S. Ali Bukhari, R. Haider, S. Admasie, Y. Oh, G. Cho, and C. Kim, "Fault detection and location in a microgrid using mathematical morphology and recursive least square methods." International Journal of Electrical Power \& Energy Systems. Vol. 102, pp. 324-331, 2018. https://doi.org/10.1016/j.ijepes.2018.04.009

[13] Dr. S. Hitesh and Dr. M. Gopal., "Kernel Recursive Least Squares Function Approximation in Game Theory Based Control." Procedia Technology. Vol. 23, pp. 264-271, 2016. https://doi.org/10.1016/j.protcy.2016.03.026

[14] A. Africa, "A Mathematical Fuzzy Logic Control Systems Model Using Rough Set Theory for Robot Applications." Journal of Telecommunication, Electronic and Computer Engineering. Vol. 9, No. 2-8, pp. 7-11, 2017.

[15] A. Africa, "A Rough Set Based Solar Powered Flood Water Purification System with a Fuzzy Logic Model." 
ARPN Journal of Engineering and Applied Sciences. Vol. 12, No. 3, pp. 638-647, 2017.

[16] S. Gaglione, A. Angrisano, A. Innac, S. Del Pizzo, and A. Maratea, "Fuzzy logic applied to GNSS." Measurement. Vol. 136, No. 3, pp. 314-322, 2019. https://doi.org/10.1016/j.measurement.2018.12.103

[17] A. Africa, J. Aguilar, C. Lim Jr, P. Pacheco, and S. Rodrin, "Automated Aquaculture System that Regulates $\mathrm{Ph}$, Temperature and Ammonia." 9th International Conference on Humanoid, Nanotechnology, Information Technology, Communication and Control, Environment, and Management (HNICEM). 2017.

https://doi.org/10.1109/HNICEM.2017.8269494

[18] A. Africa, S. Bautista, F. Lardizabal, J. Patron, and A. Santos, "Minimizing Passenger Congestion in Train Stations through Radio Frequency Identification (RFID) coupled with Database Monitoring System." ARPN Journal of Engineering and Applied Sciences. Vol. 12, No. 9, pp. 2863-2869, 2017.

[19] MATLAB Simulink. https://www.mathworks.com/ products/simulink.html. 2019.

[20] A. Africa, A. Mesina, J. Izon, and B. Quitevis, "Development of a Novel Android Controlled USB File Transfer Hub." Journal of Telecommunication, Electronic and Computer Engineering. Vol. 9, Nos. 2-8, pp. 1-5, 2017.

[21] I. Niskanen, V. Forsberg, D. Zakrisson, S. Reza, M. Hummelgård, B. Andres, I. Fedorov, T. Suopajärvi, H. Liimatainen, and G. Thungström, "Determination of nanoparticle size using Rayleigh approximation and Mie theory." Chemical Engineering Science. Vol. 201, pp. 222-229, 2019.

https://doi.org/10.1016/j.ces.2019.02.020

[22] K. Le, "Distributions of multiuser-MIMO under correlated generalised-Rayleigh fading." Signal Processing. Vol. 150, pp. 228-232, 2018. https://doi.org/10.1016/j.sigpro.2018.04.011

[23] A. Magableh, O. Badarneh, I. Shafique Ansari and T. Aldalgamouni, "Error analysis of TAS/MRC in Rayleigh fading channel with non-Gaussian noise." Journal of the Franklin Institute. Vol. 355, No. 14, pp. 6877-6888, 2018. https://doi.org/10.1016/j.jfranklin.2018.06.019

[24] A. Africa and M. Cabatuan, "A Rough Set Based Data Model for Breast Cancer Mammographic Mass Diagnostics." International Journal of Biomedical Engineering and Technology. Vol. 18, No. 4, pp. 359-369, 2015. https://doi.org/10.1504/IJBET.2015.071010

[25] A. Africa, "A Rough Set-Based Expert System for diagnosing information system communication networks." International Journal of Information and Communication Technology. Vol. 11, No. 4, pp. 496-512, 2017. https://doi.org/10.1504/IJICT.2017.10008315

[26] A. Africa, "A Logic Scoring of Preference Algorithm using ISO/IEC 25010:2011 for Open Source Web Applications Moodle and Wordpress." ARPN Journal of Engineering and Applied Sciences. Vol. 13, No. 15, pp. 4567-4571, 2018.
[27] S. K. Sharma and R. Mehra, "LMS and RLS based Adaptive Filter Design for Different Signals." International Journal of Emerging Technologies and Engineering. Vol. 92-96, 2014.

[28] A. Africa, "A Rough Set Data Model for Heart Disease diagnostics." ARPN Journal of Engineering and Applied Sciences. Vol. 11, No.15, pp. 9350-9357, 2016.

[29] S. Singh and K. L. Yadav, "Performance Evaluation of Different Adaptive Filters for ECG Signal Processing." International Journal on Computer Science and Engineering. Vol. 2, No. 5, pp. 1880-1883, 2010.

[30] J. Dhiman, S. Ahmad, and K. Gulia, "Comparison between Adaptive filter Algorithms (LMS, NLMS, and RLS)." International Journal of Science, Engineering and Technology Research. Vol. 2, No. 5, 2013.

[31] M. Tawarish and K. Satyanarayana, "A Review on Pricing Prediction on Stock Market by Different Techniques in the Field of Data Mining and Genetic Algorithm." International Journal of Advanced Trends in Computer Science and Engineering (IJATCSE). Vol. 8, No. 1, pp. 23-26, 2019. https://doi.org/10.30534/ijatcse/2019/05812019

[32] D. Ahamad, M. Akhtar, and S. Hameed, "A Review and Analysis of Big Data and MapReduce." International Journal of Advanced Trends in Computer Science and Engineering (IJATCSE). Vol. 8, No. 1, pp. 1-3, 2019. https://doi.org/10.30534/ijatcse/2019/01812019

[33] R. Rani, D. Kumar, and N. Singh, "Design of Adaptive Noise Canceller Using RLS Filter a Review." International Journal of Advanced Research in Computer Science and Software Engineering. Vol. 2, No. 11, pp. 430-433, 2012.

[34] S. Zhao-Jun and W. Ru-Gang, "A Variable Step-Size Least-Mean-Square Adaptive Filtering Algorithm: Design and Application." International Journal of Multimedia and Ubiquitous Engineering. Vol. 11, No. 2, pp. 39-48, 2016.

https://doi.org/10.14257/ijmue.2016.11.2.06 